\title{
EFECTO DE LA INGESTA DE ZUMO DE TOMATE EN EL CONTENIDO DE LICOPENO Y ÁCIDOS GRASOS DE CADENA CORTA EN HECES DE RATAS SPRAGUE DAWLEY
}

Effect of tomato juice intake on lycopene and short chain fatty acid content in faeces of Sprague Dawley rats.

V.P. Gómez*, S. Bravo, G. Martín-Pozuelo. M. Santaella, M.J. Periago.

Departamento de Tecnología de los Alimentos, Nutrición y Bromatología. Área de Nutrición y Bromatología. Facultad de Veterinaria. Universidad de Murcia. Campus de Espinardo 30100, Murcia, España.

* Autor para correspondencia: Victoria P. Gómez, Tel.: +34 868884798 Fax: +34 868888497. E-mail: vpgomez@um.es

\section{RESUMEN}

El consumo de vegetales proporciona a la dieta una amplia variedad de compuestos bioactivos que pueden ser absorbidos en el intestino durante la digestión, o llegar al intestino grueso donde son utilizados como sustratos fermentativos por la flora colónica o se mantienen intactos ejerciendo un efecto beneficioso protector sobre las células del colon. El objetivo del presente estudio ha sido evaluar la presencia de licopeno y ácidos grasos de cadena corta (AGCC) en las heces de ratas Sprague Dawley (SD) sometidas a distintas dietas. Se seleccionaron 24 ratas machos SD adultas de 12 semanas, clasificándose en tres grupos (control, grupo A y grupo B). Los animales se introdujeron en jaulas metabólicas y fueron alimentados durante 21 días con una dieta estándar y agua ad libitum (grupo control), zumo de tomate con bajo $(2.7 \mathrm{mg} / 100 \mathrm{~g}$ ) contenido en licopeno (grupo A) y zumo de tomate con alto $(12 \mathrm{mg} / 100 \mathrm{~g}$ ) contenido en licopeno (grupo B). Diariamente se recogió la orina y las heces. Se seleccionaron las muestras de heces correspondientes al inicio y fin del estudio, determinándose en ellas el contenido de licopeno colorimétricamente y los AGCC por cromatografía gaseosa. Los resultados obtenidos mostraron que el consumo de zumo de tomate producía un aumento del contenido de licopeno en heces proporcional a la cantidad de licopeno presente en el zumo. La presencia de licopeno en heces nos indica que no todo el licopeno presente en la dieta es absorbido. En relación al perfil de AGCC en heces se observa un cambio desde el inicio al final del estudio, destacando un aumento en el ácido butírico, consecuencia de la modificación en los substratos de fermentación asociada al cambio de alimentación de las ratas que tomaron zumo de tomate. La acción antioxidante del licopeno a su paso por el intestino grueso hasta su excreción en las 
heces y la formación de ácido butírico, podrían ejercer una acción beneficiosa en las células del colon, pudiéndose prevenir posibles procesos cancerígenos.

Palabras clave: tomate, licopeno, ácidos grasos de cadena corta, heces, ratas Sprague Dawley.

\section{ABSTRACT}

A wide range of bioactive components are provided to the diet by vegetables intake, which can be absorbed by the intestinal cells during digestion process. Furthermore, some of these components are susceptible to be used as fermentative substrate by intestinal microbiota as well as playing a protective role on colon cells when maintain their original structure. The aim of this research was to evaluate the content of lycopene and short-chain fatty acids (SCFA) in faeces of Sprague Dawley (SD) rats. Twenty four 12 weeks old male SD rats, were selected and classified in three different groups (Control, Group A and Group B). The animals were introduced in metabolic cages and fed during a period of 21 days using a standard diet. Control Group was given ad libitum access to food and water, whereas Group B and Group A were given tomato juice with high (12 $\mathrm{mg} / 100 \mathrm{~g})$ and low $(2.7 \mathrm{mg} / 100 \mathrm{~g})$ lycopene content, respectively. Urine and faeces were picked up everyday. Both, faeces samples at the beginning and at the end of the research period were selected, analyzing lycopene content by colorimetric method and SCFA by gas-liquid chromatography. The results showed that tomato juice intake produces an increase in the amount of lycopene in faeces. This increment was directly proportional to the lycopene content in different juices. The presence of lycopene in faeces suggests that not all the lycopene ingested was absorbed. A change in the SCFA profile in faeces has been observed from the beginning to the end of the study. In this way, an increase in butyric acid level was detected in those rats which took tomato juice. This finding could be a consequence of the modification of substrates by fermentation. The antioxidant effect of lycopene through the large intestine until its excretion and the formation of butyric acid, could be able to make a positive effect on colon cells and, eventually, prevent carcinogenic processes.

Key words: Tomato, lycopene, short-chain fatty acids, faeces, Sprague Dawley rats.

\section{INTRODUCCIÓN}

La alimentación humana ha pasado de una nutrición adecuada, donde primaba el interés por conseguir un abastecimiento suficiente $\mathrm{y}$ seguro de alimentos para garantizar la supervivencia, a una nutrición óptima basada en la optimización de la calidad de la ingesta diaria en términos de nutrientes y no nutrientes (Ashwell, 2002), presentando una mayor atención a las propiedades de los alimentos que favorecen el mantenimiento de la salud (SánchezMuniz, 2005). Dentro de esta nutrición óptima se englobaría al grupo de los alimentos funcionales, definidos como aquellos alimentos que ejercen un efecto beneficioso sobre una o más funciones selectivas del organismo, además de sus efectos nutritivos intrínsecos, de modo que resulten apropiados para mejorar el estado de salud y bienestar y reducir el riesgo de enfermedad (Diplock et al., 1999; Ashwell, 2002).
Se ha visto que el estrés oxidativo es uno de los principales factores etiológicos de la generación de las enfermedades crónicas que afectan al ser humano, provocado por la alta producción de especies reactivas de oxigeno (EROS) que causan daño oxidativo en importantes biomoléculas como los lípidos, oxidando los ácidos grasos insaturados, proteínas, aminoácidos y ADN. Estos daños oxidativos en la estructura de las biomoléculas, de no ser reparados, se acumulan en las células e incrementan el riesgo de que se presenten enfermedades crónicas (Rao \& Ali, 2007; Waliszewski \& Blasco, 2010), como enfermedades inflamatorias, cáncer, Alzheimer, cataratas o disminución funcional por envejecimiento (Liu \& Felice, 2007).

Diversas evidencias científicas sugieren que el consumo de prebióticos, como la fibra, y de antioxidantes en nuestra dieta ejercerían una acción positiva sobre nuestro bienestar, en virtud de su habilidad de interactuar con las EROS 
(Boza, 2003). Este hecho ha llevado a la Organización Mundial de la Salud a recomendar el consumo de cinco raciones de verduras y/o frutas diariamente.

Las verduras presentan multitud de compuestos bioactivos, entre los que destacaremos los carotenoides, pigmentos naturales sintetizados por plantas y microorganismos, responsables en parte del color de los mismos (Clinton, 1998; Periago et al., 2001), los cuales contribuyen a un aporte importante de antioxidantes a nuestra dieta.

De los más de cincuenta carotenoides presentes en los alimentos, destacaremos el licopeno, carotenoide de estructura acíclica, a partir del cual se sintetizan otros de gran importancia (Figura 1. Fuente: Khachick et al., 2002; Perera \& Mei Yen, 2007). El licopeno, aunque no posee acción provitamina $\mathrm{A}$, presenta un gran poder antioxidante al tener la capacidad de neutralizar el oxígeno simple o singlete $\left({ }^{1} \mathrm{O}_{2}\right)$, altamente reactivo frente a los lípidos poliinsaturados (Holger \& Ortiz, 2001), teniendo dos veces mayor poder antioxidante que el $\beta$-caroteno y cien veces mayor que la vitamina A (Boza, 2003). Algunos autores estudiaron el mecanismo de acción del licopeno y vieron que actuaba como un antioxidante (Heber \& Lu, 2002; Wertz et al., 2004). El licopeno es capaz de atrapar especies reactivas de oxígeno (EROS) y disminuir así el estrés oxidativo, reduciendo el peligro de oxidación de los componentes celulares, incluyendo lípidos, proteínas y ADN (Agarwal \& Rao, 2000), actuando como un potente antioxidante y reduciendo el riesgo de padecer las enfermedades derivadas del daño oxidativo (Wertz et al., 2004; Basu \& Imrahn, 2007), como son osteoporosis y otros desórdenes óseos, enfermedades cardiovasculares, diversos cánceres como el de próstata (Giovannucci, 1999; Wertz et al., 2004), hipertensión, enfermedades neurodegenerativas e infertilidad masculina (Waliszewski \& Blasco, 2010). La ingesta diaria de 7-10 mg de licopeno es suficiente para mantener los niveles de licopeno

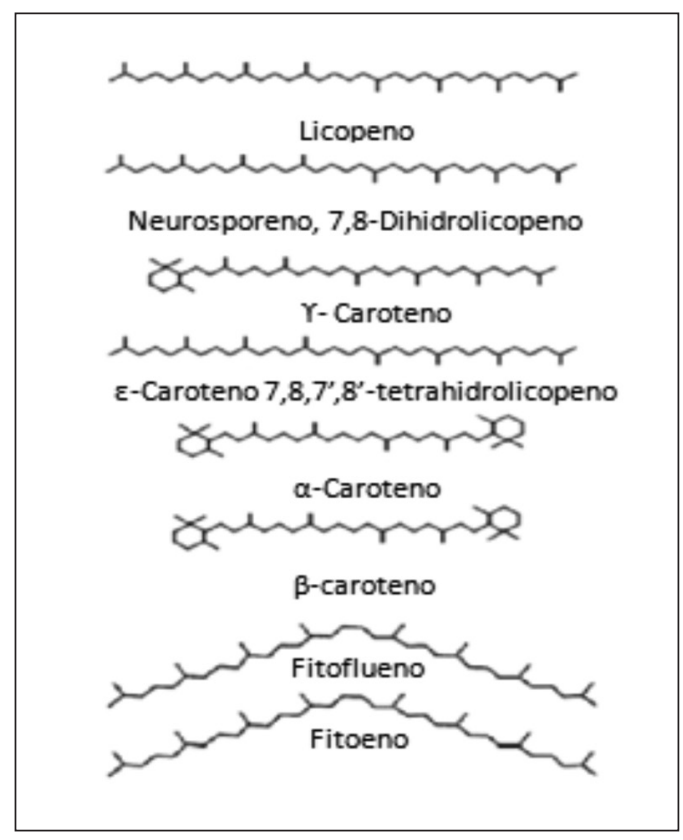

Figura 1. Estructuras químicas de carotenoides en tomates y productos a base de tomate

Fuente: Khachick et al. (2002).

necesarios para mostrar su capacidad antioxidante y prevenir enfermedades crónicas (Cámara, 2011; Rao, 2006 b).

Como fuente principal de licopeno en la dieta tenemos el tomate (Licopersicum esculentum) y productos derivados del mismo (Clinton, 1998; Rao et al., 1998; Nguyen \& Schwartz, 1999; Rao et al., 2006 a), aunque existen otros alimentos que presentan cantidades óptimas como se muestra en la Tabla 1 adaptada de Periago et al. (2001), Rao et al. (1998) y Rao et al. (2006 a). Además el consumo de tomate aporta a la dieta fibra dietética, presentando un contenido medio de $1.4 \mathrm{~g} / 100 \mathrm{~g}$ de tomate fresco (Mataix, 2002; García-Peris, 2004), de la cual el $24.3 \%$ corresponde a fibra dietética soluble (Nelly, 2000) expresado en porcentaje de peso seco y destacando en el tomate el contenido en oligosacáridos como los fructanos (De Haro, 


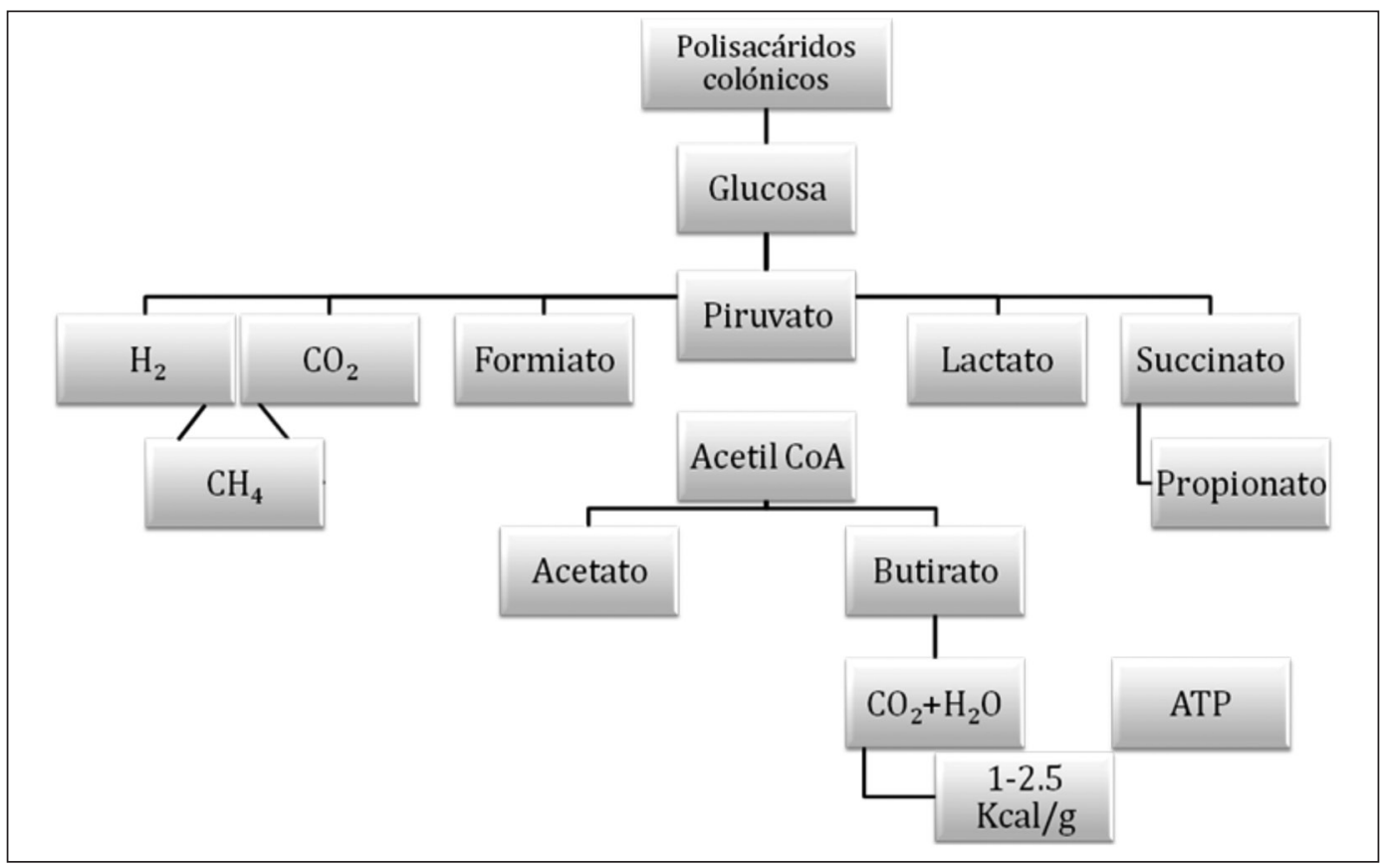

Figura 2. Productos de fermentación colónica.

Fuente: García-Peris et al. (2002).

2007), teniendo estos la característica de ser fibras con alto grado de fermentación (GarcíaPeris, 2004).

Hoy en día está reconocido el efecto beneficioso de la fibra dietética ya que se ha visto que existe una relación entre ingesta de fibra y prevención de distintas enfermedades, entre ellas cáncer de colon (Kawaguchi, 2005; Boza, 2003; Ruiz-Roso \& Pérez-Olleros, 2010). Los efectos que a nivel de proliferación bacteriana genera la fibra podrían conferirle propiedades prebióticas (García et al., 2008). Además incrementa el volumen de las heces envolviendo así posibles sustancias cancerígenas, eliminándolas más rápidamente del intestino por su poder laxante, antes de que puedan generar problemas en el organismo (Boza, 2003).

Una característica muy importante de la fibra soluble es su degradación por parte de la flora intestinal anaeróbica (Figura 2. Fuente: García-Peris et al., 2002). Este proceso da lugar a la formación de hidrógeno, dióxido de carbono, metano y ácidos grasos volátiles de cadena corta, de los cuales el $85 \%$ corresponden a acético, propiónico y butírico, en una proporción molar casi constante de 60:25:15, respectivamente (García-Peris, 2004).

Estos AGCC son resultado del metabolismo del piruvato producido por la oxidación de la glucosa a través de la vía glucolítica de Embden-Meyerhof (Wollin \& Miller, 1983; García-Peris et al., 2002; García-Peris, 2004). La proporción de AGCC que es absorbida representa el sustrato energético fundamental del colonocito (García-Peris et al., 2002). Los efectos de estos AGCC son diversos y actúan en distintos niveles sistémicos. El propionato es utilizado principalmente por el hígado en la 
Tabla 1. Contenido en licopeno de distintos alimentos

\begin{tabular}{|l|c|}
\hline \multicolumn{1}{|c|}{ FUENTE } & LICOPENO $(\mathbf{m g} / \mathbf{1 0 0}$ g alimento en fresco $)$ \\
\hline Tomate fresco & $0.72-20$ \\
\hline Zumo de tomate & $5.00-1.60$ \\
\hline Salsa de tomate & 6.2 \\
\hline Pasta de tomate & 365 \\
\hline Sopa de tomate & 7.99 \\
\hline Kétchup & $9.90-13.44$ \\
\hline Salsa para pizza & 1.71 \\
\hline Sandia & $2.3-7.20$ \\
\hline Guayaba rosa & $5.23-5.50$ \\
\hline Pomelo rosa & 3.36 \\
\hline Toronja & $0.35-3.36$ \\
\hline Papaya & $0.11-5.3$ \\
\hline Albaricoque deshidratado & 0.86 \\
\hline Zanahoria & $0.65-0.78$ \\
\hline Calabaza & $0.38-0.46$ \\
\hline
\end{tabular}

Adaptada de Periago et al. (2001), Rao et al. (1998) y Rao et al. (2006 a).

gluconeogénesis (Rombeau \& Kripke, 1990). El acetato se utiliza en la lipogénesis (Edwards, 1994), siendo el único que llega a los tejidos periféricos, principalmente el muscular, donde es metabolizado (Skuches et al., 1979; Kawaguchi, 2005). El butirato es el que presenta mayor efecto trófico sobre la mucosa intestinal (Roediger, 1982; García-Peris et al., 2002) teniendo una gran importancia, no sólo por estimular la proliferación y diferenciación de los colonocitos sanos, sino porque se ha descrito que el butirato podría ejercer un importante papel como inhibidor en el crecimiento de colonocitos neoplásicos (Velázquez et al., 1996; Escudero \& González, 2006; De Haro, 2007; Scharlau et al., 2009). Esta acción sobre las células del colon es conocida como "efecto paradójico del butirato" (Redondo, 2002; De Haro, 2007) pudiéndose explicar con ello la posible relación entre in- gesta de fibra y menor incidencia de cáncer de colon (Kawaguchi, 2005).

Como se ha mencionado el consumo de vegetales proporciona a la dieta una amplia variedad de compuestos bioactivos que pueden ser absorbidos durante la digestión o llegar al intestino grueso, donde son utilizados como sustratos fermentativos o bien manteniéndose intactos ejerciendo un efecto beneficioso protector sobre las células del colon (Pupponen-Pimiä et al., 2002). Por ello el objetivo del presente trabajo ha sido determinar la presencia de licopeno y AGCC en heces de rata, para observar posibles modificaciones en la respuesta del organismo ante la ingesta de derivados del tomate con alto y bajo contenido en licopeno, así como valorar el efecto que el consumo de fibra procedente de derivados de tomate pudiera tener en la producción de AGCC a nivel del colon. 


\section{MATERIAL Y MÉTODOS}

Las muestras de heces de rata que fueron analizadas en este trabajo, procedían de un estudio in vivo que se realizó con ratas Sprague Dawley para ver el efecto que el consumo de zumo de tomate podía tener en el perfil lipídico y en la actividad de la Hidroximetil-glutaril Coenzima A Reductasa (HMG Co A Reductasa) hepática. Dicho estudio fue llevado a cabo en el Departamento de Tecnología de Alimentos, Nutrición y Bromatología de la Facultad de Veterinaria en la Universidad de Murcia, contando con la aprobación previa del Comité de Bienestar animal de la Universidad de Murcia. En este estudio de 21 días de duración, se utilizaron 24 ratas macho de 12 semanas con un peso medio de $400 \mathrm{~g}$, suministradas por el Animalario de la Universidad de Murcia. Las ratas (4 por jaula) se mantuvieron durante 2 días en unas condiciones controladas semejantes a las que tendrían durante el estudio posterior $\left(22^{\circ} \mathrm{C}\right.$ de temperatura, $50 \%$ de humedad y ciclos de 12 horas de luz y oscuridad). En estas condiciones todas las ratas fueron alimentadas con una dieta estándar y agua ad libitum. Tras la fase de adaptación los animales se distribuyeron en jaulas metabólicas diferenciándolos en 3 grupos de 8 ratas cada uno (control, grupo A y grupo B). A todos los grupos se les suministró en comederos una dieta estándar ad libitum a base de pienso triturado Teclat Global 14\% Rodent Maintenance Diet, cuya composición en principios inmediatos y energía aparece en la Tabla 2. La diferencia entre grupos fue el tipo de bebida administrada ad libitum en biberones graduados, siendo agua para el grupo control y zumo de tomate, con bajo $(2.7 \mathrm{mg} / 100 \mathrm{~g})$ y alto $(12 \mathrm{mg} / 100 \mathrm{~g})$ contenido en licopeno, para los grupos A y B, respectivamente. Diariamente se recogieron datos de la cantidad de pienso y líquido ingerido, así como de las excreciones líquidas y sólidas de los animales. Estas tareas se vieron facilitadas por el uso de jaulas metabólicas (Techniplastgazzada, Bugugiatte, Italia) que permitieron separar la orina de las heces en recipientes diferentes. Las muestras de heces fueron envasadas en bolsas herméticas y depositadas en cajas de

Tabla 2. Composición media de principios inmediatos y energía de la dieta suministrada a las ratas en el estudio

\begin{tabular}{|l|c|}
\hline \multicolumn{1}{|c|}{ PARÁMETRO } & CONCENTRACIÓN \\
\hline Proteínas & $14.5 \%$ \\
\hline Fibra & $4.5 \%$ \\
\hline Cenizas & $4.7 \%$ \\
\hline Hidratos de carbono & $63.9 \%$ \\
\hline Almidón & $44.5 \%$ \\
\hline Azúcares & $5.7 \%$ \\
\hline Grasas & $4.0 \%$ \\
\hline De las cuales: & $7.2 \%$ \\
Ácidos grasos saturados & $8.3 \%$ \\
Ácidos grasos monoinsaturados & $24.9 \%$ \\
\hline Ácidos grasos poliinsaturados & $3.2 \mathrm{Kcal} / \mathrm{g}$ \\
\hline Energía digerible & $3.1 \mathrm{Kcal} / \mathrm{g}$ \\
\hline Energía metabolizable & \\
\hline
\end{tabular}


poliestireno expandido recubiertas de hielo para su traslado hasta un congelador de $-80^{\circ} \mathrm{C}$, donde permanecieron hasta el día de su procesado para el análisis. En el ensayo se tomaron otras muestras biológicas, que no han sido utilizadas en el presente estudio.

\section{Análisis del contenido en licopeno}

Para determinar el contenido en licopeno se empleó el método descrito por Sharma \& Le Maguer (1996), modificando la cantidad de muestra a tomar y el tiempo de agitación en la fase extractiva por la dificultad encontrada para disolver las muestras de heces. Para realizar la extracción del licopeno, a partir de las muestras previamente descongeladas, se pesó $1 \mathrm{~g}$ de heces al que se adicionaron $50 \mathrm{ml} \mathrm{de}$ la mezcla extractiva hexano-acetona-etanol (2:1:1, v:v:v). La mezcla se mantuvo en una placa de agitación magnética y tras 30 minutos se le añadieron $15 \mathrm{ml}$ de agua destilada, volviendo a agitar y consiguiendo la separación de fase polar y no polar (en la que se encontraba el licopeno extraído). La fase no polar fue filtrada a través de un filtro Whatman $n^{\circ}$ 1 , procediendo a su cuantificación en un espectrofotómetro Thermo Scientific Evolution 300LC (England) en el que se fijó una longitud de onda de $472 \mathrm{~nm}$ apropiada para la lectura de absorbancia. Para el cálculo de la concentración del licopeno consideramos el coeficiente de extinción molar ( $\varepsilon 1 \%$ ), siendo para el licopeno a una longitud de onda de $472 \mathrm{~nm}$, de $3450 \mathrm{~cm}^{-1}$ (Sharma \& Le Maguer, 1996). El valor de absorción aparente de licopeno se obtuvo aplicando la siguiente fórmula: (I - E / I) x 100; siendo: I, el licopeno ingerido y E, el licopeno excretado en heces.

\section{Análisis del perfil de ácidos grasos de cadena corta}

Para conocer el perfil de AGCC en las heces de las ratas, partimos del método descrito por
Girard-Pipau et al. (2002), modificando, tanto la cantidad de partida de muestra de heces, como los tiempos de agitación, debido a la dificultad que encontramos para la homogeneización de las muestras.

La preparación de la muestra, a la que posteriormente se le realizaría su análisis cromatográfico, consistió en pesar en un tubo de cristal Pyrex $400 \mathrm{mg}$ de heces, previamente descongeladas, a las que se añadieron $1.8 \mathrm{ml}$ de agua destilada, sometiéndolas a agitación hasta conseguir una suspensión lo más homogénea posible. Posteriormente, se adicionaron $0.4 \mathrm{ml}$ de ácido sulfúrico al $50 \%$ en agua, $0.8 \mathrm{~g}$ de $\mathrm{NaCl}$ y $1 \mathrm{ml}$ de dietil éter (cada vez que se añadía un reactivo se homogeneizaba el contenido del tubo en un vortex Heidolph Reax 2000, Germany). Después se procedió a la fase extractiva que consistió en colocar los tubos Pyrex en un agitador orbital OVAN modelo N12-E (EU) durante 1 hora, llevándolos después a una centrífuga Nahita modelo 2690 a 1207 x g (3000 rpm) durante 5 minutos. Para comenzar la etapa analítica se trasvasó el sobrenadante a un vial de 0.2 ml Chromacol de Supelco (USA) con tapón con septum, para su inyección en un cromatógrafo de gases 7890A GC System de Agilent Technologies (Santa Clara, USA), con detector de ionización de llama, inyector automático y una columna capilar Nukol ${ }^{\text {TM }}$ (ref. 24107, USA) de $30 \mathrm{~m}$ de longitud, $0.25 \mathrm{~mm}$ de diámetro interno y $0.25 \mu \mathrm{m}$ de espesor de película. Como gas portador se empleó Helio siendo la presión del inyector constante de $58.99 \mathrm{kPa}$. La rampa de temperatura del horno fue la siguiente: $80^{\circ} \mathrm{C}$ durante 5 minutos $/ 5^{\circ} \mathrm{C} / \mathrm{min}$ hasta $185^{\circ} \mathrm{C}$. La temperatura del detector fue de $220^{\circ} \mathrm{C}$, la del inyector de $220^{\circ} \mathrm{C}$ y el flujo de aire y de hidrógeno de $400 \mathrm{ml} / \mathrm{min}$ y $30 \mathrm{ml} / \mathrm{min}$, respectivamente. Para la identificación de los distintos AGCC se utilizó un multipatrón comercial con una concentración de $10 \mathrm{mM}$ (SUPELCO, ref. 46975-U, USA) de ácidos grasos de cadena corta de calidad y composición certificada (ácido acético, ácido propiónico, ácido isobutírico, 
ácido butírico, ácido isovalérico, ácido valérico, ácido isocapróico, ácido capróico y ácido heptanóico) a partir del cual se prepararon multipatrones $1 \mathrm{mM}, 5 \mathrm{mM}$ y $10 \mathrm{mM}$. Debido a que nuestras muestras presentaban una cantidad mayor de ácido acético y ácido propiónico, se prepararó además un multipatrón con una cantidad de $0.12 \mathrm{~g}$ de ácido acético y $0.15 \mathrm{~g}$ de ácido propiónico, llevando el volumen a $10 \mathrm{ml}$, consiguiendo así una disolución madre de 199 mM de ácido acético y de 201.6 mM de ácido propiónico a partir de las cuales obtuvimos las disoluciones de trabajo. Todos los patrones utilizados para realizar la recta patrón fueron procesados igual que el resto de las muestras, realizando la calibración con estándar externo.

\section{Análisis estadístico}

Los datos obtenidos fueron tratados mediante el paquete SPSS 15.0. Se llevó a cabo un análisis descriptivo, expresando los datos en forma de valor medio, error típico y desviación estándar según parámetro. Para los AGCC se aplicó un análisis de varianza (ANOVA), empleando un intervalo de confianza del 95\% (considerando diferencias estadísticamente significativas cuando $\mathrm{p}<0.05)$ y un test de separación de medias (HSD de Tukey) en los casos en que se rechazó la hipótesis de igualdad de medias. También se realizó una comparación de medias aplicando una $\mathrm{T}$ de Student para las muestras inicio y final de cada grupo. En el caso del contenido de licopeno en heces, además del análisis descriptivo, se llevó a cabo una correlación de Pearson junto con el contenido de AGCC en heces, considerando diferencias estadísticamente significativas cuando $\mathrm{p}<0.05$.

\section{RESULTADOS Y DISCUSIÓN}

\section{Evolución del peso de las ratas}

En los grupos control y $\mathrm{B}$ se produjo un incremento medio de ganancia de peso de $8.4 \%$, mientras que el grupo A sólo aumentó un 1.5\%. Las cantidades ingeridas de alimento y bebida, junto con las excreciones recogidas, mostradas en la Tabla 3 se relacionaron con este incremento. Estos datos ponen de manifiesto que el consumo de zumo de tomate con alto contenido en licopeno no modifica el apetito en las ratas. Sin embargo, el zumo de tomate con bajo contenido en licopeno y con características organolépticas distintas afecta a la ingesta, tanto de alimento, como de bebida.

\section{Contenido fecal de licopeno}

En relación con el licopeno, se muestra que ambos grupos presentaron niveles de excreción fecal muy similares. El consumo medio de licopeno (Tabla 3) fue de $0.5 \mathrm{mg} /$ día para el grupo al que se le suministró el zumo A y de $3.6 \mathrm{mg} /$ día para el grupo que ingirió el zumo B. El licopeno en heces también fue analizado para los dos tipos de zumos suministrados tanto al inicio como al final del estudio, obteniéndose valores que evolucionaron desde 0 a $0.1 \mathrm{mg} /$ día para los individuos del grupo al que se les suministró el zumo A y de 0.03 a 0.2 para los del zumo B. Los datos de absorción aparente (ver Tabla 3) fueron del 100\% y $93.7 \%$ (inicio y final, respectivamente) en el caso del grupo A, y del $96.7 \%$ y $93 \%$ (inicio y final, respectivamente) en el grupo B.

Con el estudio se pudo determinar un aumento en el contenido de licopeno presente en las heces directamente proporcional a la cantidad del mismo en el zumo suministrado, por lo que la excreción de licopeno fue mayor en aquellos individuos que ingirieron zumo con alto contenido en licopeno. Estos datos concuerdan con los anteriormente obtenidos por Schnäbele et al. (2008), donde se puso de manifiesto que el consumo de zumo de tomate incrementaba los niveles de licopeno en heces humanas. 
Tabla 3. Valor medio y desviación estándar obtenidos en diferentes parámetros relacionados con la ingesta

\begin{tabular}{|l|l|l|l|}
\hline & \multicolumn{1}{|c|}{ Control } & \multicolumn{1}{|c|}{ Zumo A } & \multicolumn{1}{c|}{ Zumo B } \\
\hline Ingesta media de comida (g/día) & $22.2 \pm 4.3$ & $18.2 \pm 4.8$ & $22.7 \pm 5.1$ \\
\hline Ingesta media de bebida (ml/día) & $20.4 \pm 3.5$ & $17.9 \pm 10.4$ & $30.1 \pm 6.9$ \\
\hline Excreción urinaria media (ml/día) & $9.6 \pm 2.5$ & $6.1 \pm 2.2$ & $14.3 \pm 4.4$ \\
\hline Excreción fecal media (g/día) & $9.3 \pm 1.4$ & $7 \pm 1.4$ & $8.9 \pm 1.1$ \\
\hline Consumo medio de licopeno (mg/día) & & $0.5 \pm 0.3$ & $3.6 \pm 0.8$ \\
\hline Licopeno medio en heces (mg/día) Inicio & & 0 & $0.1 \pm 0.02$ \\
\hline Licopeno medio en heces (mg/día) Final & & $0.03 \pm 0.01$ & $0.2 \pm 0.03$ \\
\hline Absorción aparente de licopeno(\%) Inicio* & & 100 & 96.7 \\
\hline Absorción aparente de licopeno(\%) Final* & & 93.7 & 93 \\
\hline
\end{tabular}

* El valor de absorción aparente de licopeno se obtuvo aplicando la siguiente fórmula: (I - E / I) x 100; siendo: I, el licopeno ingerido y E, el licopeno excretado.

\section{ÁCIDOS GRASOS DE CADENA CORTA EN HECES}

En cuanto a los ácidos grasos de cadena corta se halló en el grupo control un valor total de $49.2 \mathrm{mmol} /$ litro $(\mathrm{mM})$ y $80.6 \mathrm{mM}$ correspondientes con la fase inicial y final del estudio, respectivamente. De estos valores totales el $91.5 \%$ y $89.2 \%$ se correspondieron con el ácido acético, el $4.3 \%$ y $5.7 \%$ al ácido propiónico y el $2.3 \%$ y el $2.4 \%$ con el ácido butírico, en las fases inicial y final, respectivamente.

En lo que respecta al grupo A, se obtuvo un valor total inicial de $35.9 \mathrm{mM}$ y un valor final de $76.8 \mathrm{mM}$, siendo la proporción para el ácido acético de $92.2 \%$ y $88.4 \%, 4.1 \%$ y $6.0 \%$ para el ácido propiónico, y de $1.3 \%$ y $3.4 \%$ para el ácido butírico, correspondientes a las fases inicial y final, respectivamente.

En el grupo B los valores totales de AGCC en la fase inicio fue de $51.7 \mathrm{mM}$ y $100.6 \mathrm{mM}$ en la fase final, la proporción para los AGCC mayoritarios fue de $90.8 \%$ y $85.6 \%$ de ácido acé- tico, $5.3 \%$ y $6.2 \%$ de ácido propiónico y $2.2 \%$ y $4.9 \%$ de ácido butírico, correspondientes a las fases inicio y final del estudio, respectivamente.

En cuanto a los valores de ácido butírico recogidos en heces al inicio y al final del estudio, no se encontraron diferencias estadísticamente significativas $(\mathrm{p}<0.05)$, en el caso del grupo control (Figura 3), sí hallándose en los grupos A y B, que consumieron zumo de tomate (Figuras 4 y 5). Estos resultados pueden explicarse por el aporte de fibra soluble procedente de los distintos zumos de tomate usados en el estudio. El zumo A fue elaborado a partir de tomates tipo "ensalada", que presentan un aporte en fibra soluble menor que los tomates tipo "industrial" (Nelly, 2000), empleados para la elaboración del zumo B.

A mayor contenido en fibra soluble mayor grado de fermentación en el colon, lo que conllevaría un aumento en la producción de ácido butírico, tal y como observaron Edwards et al. (1992), con el consecuente beneficio en la salud colónica (Redondo, 2002; Beyer-Sehlmeyer, 
GRUPO CONTROL

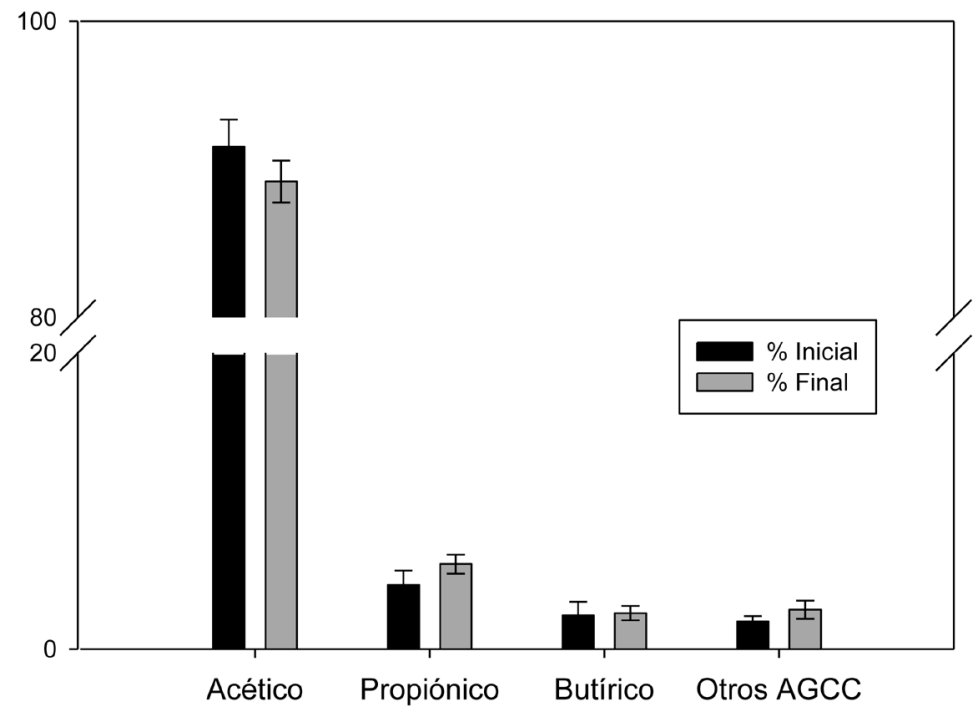

Figura 3. Porcentaje medio y error típico de los ácidos grasos de cadena corta al inicio y al final determinados en heces de rata Sprague Dawley del grupo control.

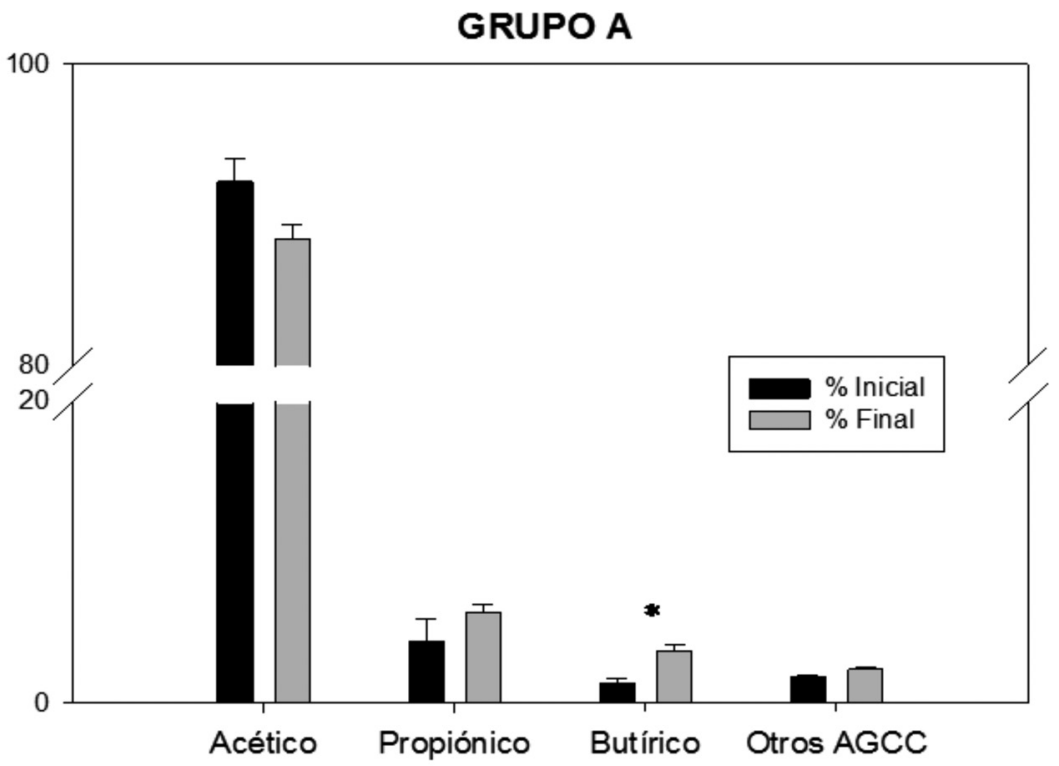

Figura 4. Porcentaje medio y error típico de los ácidos grasos de cadena corta al inicio y al final determinados en heces de rata Sprague Dawley del grupo A.

* Indica diferencias estadísticamente significativas ( $\mathrm{p}<0.05$ significativo al 95\%). 


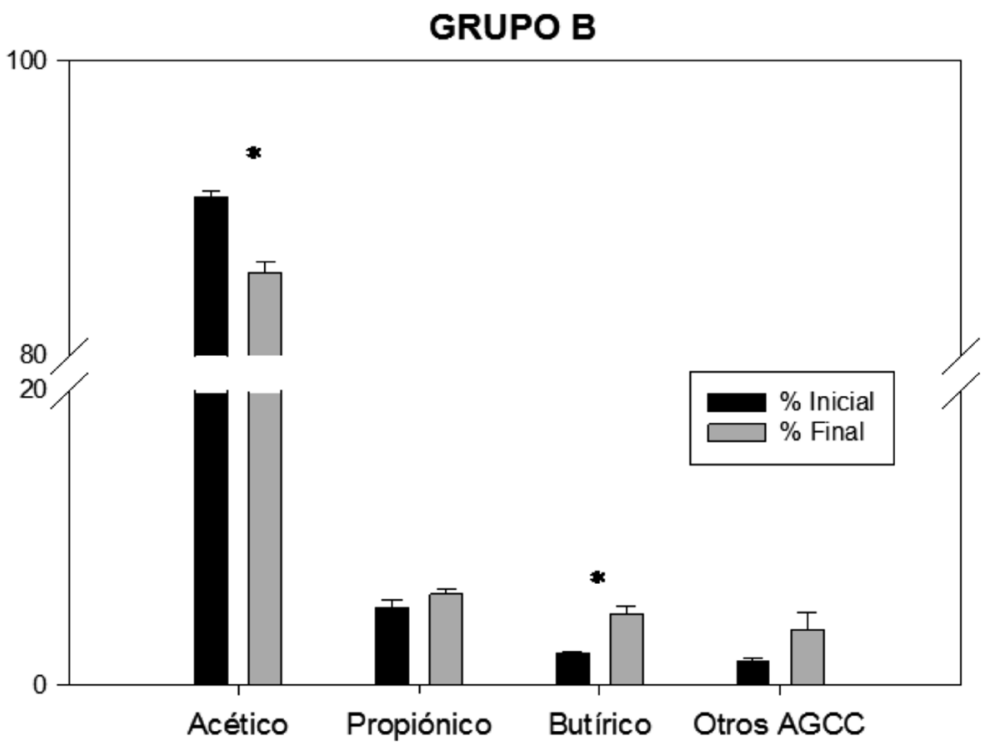

Figura 5. Porcentaje medio y error típico de los ácidos grasos de cadena corta al inicio y al final determinados en heces de rata Sprague Dawley del grupo B.

* Indican diferencias estadísticamente significativas ( $\mathrm{p}<0.05$ significativo al 95\%).

2003; Escudero \& González, 2006; De Haro, 2007; Scharlau et al., 2009). Aun existiendo diferencias en los porcentajes de ácido butírico final de los grupos A y B, no aparecen diferencias significativas $(p>0.05)$ entre estos grupos, pero sí entre el grupo control y el grupo B $(\mathrm{p}<0.05)$, posiblemente asociado a una mayor ingesta de fibra en este segundo grupo. A nivel intragrupal, encontramos diferencias estadísticamente significativas entre el inicio y el fin del estudio para el ácido butírico en el caso del grupo A, y para el ácido acético y butírico en el caso del grupo B. No existieron diferencias significativas para el grupo control.

Tras observar en el grupo B un incremento del contenido de ácido butírico en heces directamente proporcional al contenido de licopeno encontrado en heces, aplicamos una correlación de Pearson para ver el posible efecto de la ingesta de licopeno a diferentes dosis en la producción de AGCC en heces, resultando esta correlación positiva $(r=0.556 ; p<0.05)$ entre las variables ácido butírico y contenido de licopeno en heces.

\section{CONCLUSIONES}

Podemos concluir que una ingesta frecuente de zumo de tomate sumaría dos efectos beneficiosos para el organismo y concretamente para la salud del colon, como son la acción antioxidante del licopeno a su paso por el intestino hasta su excreción en las heces, junto con el incremento de los niveles fecales de butirato, combinándose así dos factores muy importantes para conseguir una buena salud intestinal.

\section{BIBLIOGRAFÍA}

AGARWAL, S., RAO, V. A. 2000. Tomato lycopene and its role in human health and chronic diseases. Canadian Medical Association Journal. 163:739-744. 
ASHWELL, M. 2002. Concepts of functional foods. International Life Science Institute. (ILSI) Europe Concise Monograph Series. Brussels.

BASU, A., IMRAHN, V. 2007. Tomatoes versus lycopene in oxidative stress and carcinogenesis: conclusions from clinical trials. European Journal of Clinical Nutrition. 61 (3): 295-303.

BEYER-SEHLMEYER, G., GLEI, M., HARTMANN, E., HUGHES, R., PERSIN, C., BÖHM, V., ROWLAND, I., SCHUBERT, R., JAHREIS, G., POOL-ZOOBEL, B.L. 2003. Butyrate is only one of several growth inhibitors produced during gut flora mediated fermentation of dietary fibre sources. British of Journal of Nutrition, 90:10571070.

BOZA, J. 2003. Alimentación y enfermedad. Anales de la Real Academia de Ciencias Veterinarias de Andalucía Oriental. 16 (1): 167-200.

CÁMARA, M. 2011. Papel del licopeno en la prevención de enfermedades. Alimentación y salud. Disponible en http://www.pmfarma.es/ noticias/12728-el-ketchup-un-aliado-en-laprevencion-de-distintos-tipos-de-cancer.html

CLINTON, S. K. 1998. Lycopene: Chemistry, biology and implications for human health and disease. Nutrition Reviews. 56: 35-51.

DE HARO, O. 2007. Efecto de la fibra en un modelo de colitis experimental en rata. $\mathrm{Pa}$ pel de los ácidos grasos de cadena corta. Tesis doctoral. Universidad de Granada.

DIPLOCK, A. T., AGGETT, P. J., ASHWELL, M., BORNET, F., FERN, E. B., ROBERFROID, M. B. 1999. Scientific concepts of functional food in Europe: Consensus document. British Journal of Nutrition. 81(1):127.

EDWARDS, C.A., WILSON, R.G., HANLON, L., EASTWOOD, M.A. 1992. Effect of the dietary fibre content of lifelong diet on colonic cellular proliferation in the rat. Gut. 33:1076-1079.
EDWARDS, C. 1994. The nutritional impact of the intestinal microflora. South African Medical Journal. Suppl: 38-34.

ESCUDERO, E., GONZÁLEZ, P. 2006. La fibra dietética. Nutrición hospitalaria. 21(2):61-62.

GARCÍA-PERIS, P., BRETÓN, I., DE LA CUERDA COMPES, C., CAMBLOR, M. 2002. Metabolismo colónico de la fibra (Artículo científico en línea). Nutrición Hospitalaria. 2: 11-16.

GARCÍA-PERIS, P. 2004. La fibra en la alimentación: Ámbito hospitalario. Edikamed. Barcelona. Pp. 1-17.

GARCÍA, E., BENITO, R., RIVERA, C. 2008. Hacia una definición de la fibra alimentaria. Anales Venezolanos de Nutrición. 21 (1): 25-30.

GIOVANNUCCI, E. 1999. Tomatoes, tomatobased products, lycopene, and cancer. Review of the epidemiologic literature. Journal of the National Cancer Institute. 91:317-331.

GIRARD-PIPAU, F., POMPEI, A., SCHNEIDER, S., NANO, J. L., HEBUTERNE, X., BOQUET, P., RAMPAL, P. 2002. Intestinal microflora short chain and cellular fatty acids influence of a probiotic Saccharomyces boulardii. Microbial Ecology in Health Disease. 14: 220-227.

HEBER, H. N., LU, Q-L. 2002. Overview of mechanisms of action of lycopene. Experimental Biology and Medicine. 227:920-923.

HOLGER, M. S., ORTIZ, N. D. 2001. Efecto Antioxidante de los Micronutrientes. Archivos Venezolanos de Farmacología y Terapéutica. 20 (1): 4-5.

KAWAGUCHI, F. 2005. Nutrición y cáncer en la mujer. Sección de gastroenterología. Departamento de Medicina Interna. Facultad de Medicina. Universidad de Concepción. Chile. Conferencia. Disponible en http:// www.archivo.co.cl/pdf/fkawaguchi1.pdf.

KHACHICK, F., CARVALHO, L., BERNSTEIN, S.P., MUIR, G.J., ZHAO, D., KATZ, N.B. 2002. Chemistry, distribution, and me- 
tabolism of tomato carotenoides and their impact on human health. Experimental Biology and Medicine. 227:845-851

LIU, R.H., FELICE, D.L. 2007. Antioxidants and whole food phytochemicals for cancer prevention. Eds. Fereidoon Shahidi y ChiTang Ho. Antioxidant Measurement and Applications, American Chemical Society. Washington, D.C. Pp.15-34.

MATAIX, J. 2002. Nutrición y alimentación humana. Tomo I Nutrientes y alimentos. Ergón. Majadahonda. Madrid. Pp 579.

NELLY PAK, D. 2000. Fibra dietética en verduras cultivadas en Chile. Archivos Latinoamericanos de Nutrición. 50(1):97-101.

NGUYEN, M. L., SCHWARTZ, S. J. 1999. Lycopene: chemical and biological properties. Food Technology. 53: 38-53.

PERERA, C.O., MEI YEN, G. 2007. Functional properties of carotenoides in human health. International Journal Food Properties. 10: 201-230.

PERIAGO, M.J., MARTÍNEZ-VALVERDE, I., ROS, G., MARTÍNEZ, C., LÓPEZ, G. 2001. Propiedades químicas, biológicas y valor nutritivo del licopeno. Anales de Veterinaria (Murcia). 17: 51-66.

PUUPPONEN-PIMIÄ, R., AURA, A.M., OKSMAN-CALDENTEY, K.M., MYLLÄRINEN, P., SAARELA, M., MATTILASANDHOLM, T., POUTANEN, K. 2002. Development of functional ingredients for gut health. Trends in Food Science \&. Technology. 13: 3-11.

RAO, A. V., WASEEM, Z., AGARWAL, S. 1998. Lycopene content of tomatoes and tomato products and their contribution to dietary lycopene. Food Research International. 31: 737-741. RAO, A.V., RAY, M.R., RAO, L.G. 2006 a. Lycopene. Advances in Food and Nutrition Research. 51. Pp.737-741.

RAO, A. V. 2006 b. Tomatoes, lycopene \& Human Health, Preventing chronic diseases. Caledonian Science Press Limited. Badalona. Pp. 96-97.
RAO, A.V., ALI, A. 2007. Biologically active phytochemicals in human health: Lycopene. International Journal of Food Properties. 10: 279-288.

REDONDO, L. 2002. Propiedades de la fibra dietética. La fibra terapéutica. Glosa. Barcelona. Pp. 29-51.

ROEDIGER, W.E. 1982. The effect of bacterial metabolites on nutrition and function of the colonic mucosa. Symbiosis between man and bacteria. Falk Symposium 32. Eds. Kaspes, H., Goebell, H. Colon and Nutrition. MTP Press limited. Lancaster. Pp. 11-24.

ROMBEAU, J.L., KRIPKE, S.A. 1990. Metabolic and intestinal effects and short chain fatty acids. Journal of Parenteral and Enteral Nutrition. 14:181-185.

RUIZ-ROSO, B., PÉREZ-OLLEROS, L. 2010. Avance de resultados sobre consumo de fibra en España y beneficios asociados a la ingesta de fibra soluble. Revista Española de Nutrición Comunitaria. 16:147-153.

SÁNCHEZ-MUNIZ, F.J. 2005. Departamento de Nutrición y Bromatología de UCM. Nuevos alimentos. Realidad y perspectivas de la carne y sus derivados como alimentos funcionales. Fundación Española de Nutrición (FEN). Madrid. Pp. 43-54.

SCHNÄBELE, K., BRIVIBA, K., BUB, A., ROSER, S., POOL-ZOBEL, B.L. AND RECHKEMMER, G. 2008. Effects of carrot and tomato juice consumption on faecal markers relevant to colon carcinogenesis in humans. British Journal of Nutrition. 99: 606-613.

SCHARLAU, D., BOROWICKI, A., HABERMANN, N., HOFMANN, T., KLENOW, S., MIENE, C., MUNJAL, U., STEIN, K., GLEI, M. 2009. Review Mechanisms of primary cancer prevention by butyrate and other products formed during gut flora-mediated fermentation of dietary fibre. Mutation Research. 682: 39-53.

SHARMA, S., LE MAGUER, M. 1996. Lycopene in tomatoes and tomato pulp fractions. Italian Journal of Food Science. 2: 101-113. 
SKUCHES, C.L., HOFRYDE, C.P., MYERS, R.N. 1979. Plasma acetate turnover and oxidation. Journal of Clinical Investigation. 64:708-713.

VELÁZQUEZ, O.C., LEDERER, H.M., ROMBEAU, J.L. 1996. Butyrate and the colonocyte. Implications for neoplasia. Digestive Diseases and Sciences. 41: 727-739.

WALISZEWSKI, K. N., BLASCO, G. 2010. Artículo de revisión-Propiedades nutraceú- ticas del licopeno. Salud Pública de México. 52: 254-265.

WERTZ, K., SILER, U., GORALCZYK, R. 2004. Lycopene: Modes of action to promote prostate health. Archives of Biochemistry and Biophysics. 430:127-134

WOLLIN, M.J., MILLER, T.L. 1983. Interactions of microbial populations in cellulose fermentations. Federation Proceedings. 42: 109-113. 